\title{
Comparison of Exothermic Release during the Polymerization of Four Materials used to fabricate Provisional Restorations
}

\section{Minu Raju}

\begin{abstract}
Background and objectives: This in vitro study compared and determined the temperature increase in the pulp chamber of permanent maxillary first premolar teeth placed in contact with different resins used for the fabrication of provisional restorations.
\end{abstract}

Materials and methods: Polymethyl methacrylate (DPI), polyethyl methacrylate (SNAP), polyvinylethyl methacrylate (TRIM), bis-acrylic composite (Protemp II) were compared with respect to their exothermic properties during polymerization. Eighty freshly extracted maxillary first premolars were prepared for complete coverage restoration were placed in an acrylic resin block. A thermal probe connected to a digital thermometer was placed into the pulp chamber. Specimens were submerged in a water bath to simulate intraoral conditions. The provisional materials were manipulated according to manufacturer's instructions. The resin mix was placed into template and was then positioned on the prepared tooth. The temperature was recorded during the polymerization at 30 -second intervals until it was evident that the peak temperature had been reached.

After complete polymerization, the template was removed from the tooth and the provisional crown was retrieved. Data was analyzed with one-way ANOVA and Bonferroni multiple comparisons test.

Results: Mean temperature increase for the provisional crown fabrication ranged from $38.78^{\circ} \mathrm{C}, 37.79^{\circ} \mathrm{C}, 37.71^{\circ} \mathrm{C}$ and $35.88^{\circ} \mathrm{C}$ for polymethyl methacrylate, polyethyl methacrylate, polyvinylethyl methacrylate and bis-acrylic composite respectively.

Polymethyl methacrylate, polyethyl methacrylate and bisacrylic composite were highly significant.

Interpretation and conclusion: All the materials used were in the safer limit. Hence, by comparing all the four material, Bis-acrylic composite showed least temperature rise in pulp chamber.

Keywords: Provisional restoration, Pulp chamber, Polymerization, Acrylic resins, Specimens.

How to cite this article: Raju M. Comparison of Exothermic Release during the Polymerization of Four Materials used to

\footnotetext{
Assistant Professor

Department of Prosthodontics, Manipal College of Dental Sciences, Manipal University, Manipal, Karnataka, India

Corresponding Author: Minu Raju, Assistant Professor Department of Prosthodontics, Manipal College of Dental Sciences, Manipal, Karnataka, India, Phone: 09481214726 e-mail: drminpros31@gmail.com
}

fabricate Provisional Restorations. Int J Prosthodont Restor Dent 2014;4(1):1-5.

Source of support: Nil

Conflict of interest: None declared

\section{INTRODUCTION}

Provisional restorations are an integral part of fixed prosthodontics treatment. Major requirements of these materials are that they should be nonirritating to pulp and to other tissues and should have low exothermicity. ${ }^{1}$

Provisional restorations are used between the initiation of treatment and placement of definitive prostheses. ${ }^{2}$ Provisional restoration protect the pulp and tissue of prepared abutments, promote gingival healing, and defend the abutments from microleakage and chemical injuries. ${ }^{3}$ It also helps in stabilization of occlusion, function, esthetic enhancement and provision of diagnostic information. ${ }^{4}$

Pulpal injury during fabrication of provisional crowns may result from heat produced from polymerization of resins, desiccation or chemical insult from the monomer. ${ }^{5}$ So, precaution should be taken before the fabrication of provisional restoration, since the material may be detrimental to the pulp.

A histologic animal study has demonstrated that the healthy pulps failed to recover from an intrapulpal temperature rise of $5.55^{\circ} \mathrm{C}$ in $15 \%$ of the situations. When the intrapulpal temperature increased by $11.1^{\circ} \mathrm{C}$ and $16.65^{\circ} \mathrm{C}$, 60 and $100 \%$ of the teeth, respectively, lost vitality. ${ }^{6}$

Thus, it becomes imperative for the clinicians to be aware of increased temperature levels associated with the direct fabrication of provisional restoration and take necessary precautions to minimize iatrogenic trauma to the pulp.

Therefore, the present study is undertaken to determine and compare the temperature increase in the pulp chamber of maxillary teeth placed in contact with different resins used for the direct fabrication of provisional restorations.

\section{MATERIALS AND METHODS}

\section{Source of Data}

In vitro study was conducted in the Department of Prosthodontics including Crown and Bridge and Implantology, AB Shetty Memorial Institute of Dental Sciences, Mangalore. 
Subject requiring tooth extractions for orthodontic treatment were selected. Eighty freshly extracted permanent maxillary first premolars were collected from the department of oral and maxillofacial surgery of the same institute.

\section{Method of Collection of Data}

The study was planned as follows:

1. Sample size: 80 samples were made into four groups for this study.

2. Case group.

As a provisional restoration, polymethyl methacrylate (PMMA), polyethyl methacrylate (PEMA), polyvinylethyl methacrylate (PVEMA) and bis-acrylic (bis-A) composite were used on each 20 samples and were considered as groups I, II, III, IV respectively.

\section{Selection Criteria for Subjects}

\section{Inclusion Criteria}

Twenty freshly extracted healthy permanent premolar teeth.

\section{Exclusion Criteria}

Decayed/restored teeth, severely attrited teeth and endodontically treated teeth.

\section{Procedure}

Twenty freshly extracted maxillary teeth of young adults were stored in $1 \%$ chloramine solution for a period of 2 weeks. Each tooth was then being stabilized with a minimum amount of sticky wax in the vertical position in a $4.5 \times$ $2.5 \times 0.4 \mathrm{~mm}$ box made of boxing wax. Auto polymerizing resin was hand mixed and poured under vibration in the wax box, covering the root portion of the tooth. After complete polymerization, an irreversible hydrocolloid impression of the tooth and the resin block was made using a custom tray made of auto polymerizing resin. Dental stone (type III) was mixed under vacuum and impression was poured under vibration. Later, the template was made using VPS impression material (putty consistency) (Fig. 1).

Then tooth was prepared for a complete coverage restoration using a high speed air turbine handpiece and diamond points. The axial wall coverage was maintained using standard protocol. The reduction of the axial wall was $1.5 \mathrm{~mm}$ and the occlusal reduction was $2.0 \mathrm{~mm}$. The reduction was evaluated with the template. To access the pulp chamber from the apical side of the tooth, the pulpal tissues and apical portion of the teeth were removed.

The pulp chamber was then cleaned of all organic remnants using a 5.25\% sodium hypochlorite. A thermal probe connected to a digital thermometer (Fig. 2) was placed into the pulp chamber, touching the roof of the pulp chamber.
Amalgam (Fig. 3) was then condensed into the pulp chamber, surrounding and stabilizing the thermal probe in position. Specimens were submerged in water bath containing distilled water at a temperature of $36^{\circ} \mathrm{C}$ to simulate intraoral conditions.

The four provisional restorative materials used in the study were PMMA (Fig. 4), PEMA (Fig. 5), PVEMA (Fig. 6) and bis-A (Fig. 7).

Each one was measured and mixed according to the manufacturer's instructions. The template was completely filled with one of the resins and then positioned on the prepared tooth. The temperature was recorded during polymerization at 30 seconds interval, until no further increase in temperature is noted. After complete polymerization, the template was removed from the tooth and the provisional crown was retrieved. The procedure was repeated with the remaining provisional restorative resin materials. Data was analyzed with descriptive statistics and one-way analysis of variance (ANOVA).

\section{RESULTS}

Polymethyl methacrylate shows the highest mean temperature increase in pulp chamber during the polymerization of all the four tested provisional materials fabricated by direct tech-nique followed by PEMA, PVEMA and bis-A composite (Graph 1).

Table 1 shows the mean value temperature obtained were $38.785^{\circ} \mathrm{C}, 37.794^{\circ} \mathrm{C}, 37.718^{\circ} \mathrm{C}$, and $35.882^{\circ} \mathrm{C}$ for PMMA, PEMA, PVEMA and bis-A composite respectively.

The statistical analysis showed highly significant when mean difference of PMMA was compared with mean difference of PEMA, PVEMA and bis-A composite and the mean differences were $0.9910,1.067$, and 2.903 respectively (Table 2).

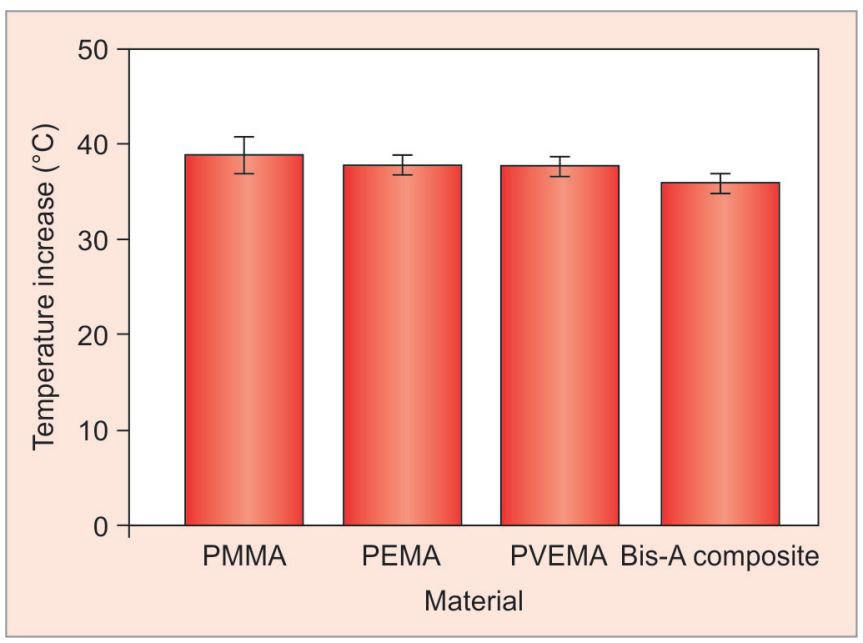

Graph 1: Comparison of mean temperature increase in pulp chamber during the polymerization of all the four tested provisional materials fabricated by direct technique 


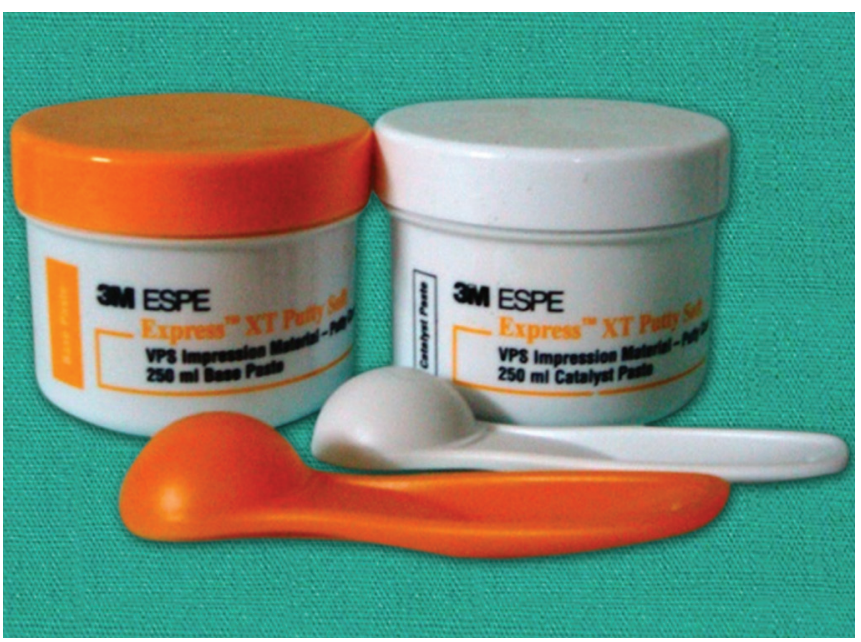

Fig. 1: Template: Addition silicone putty

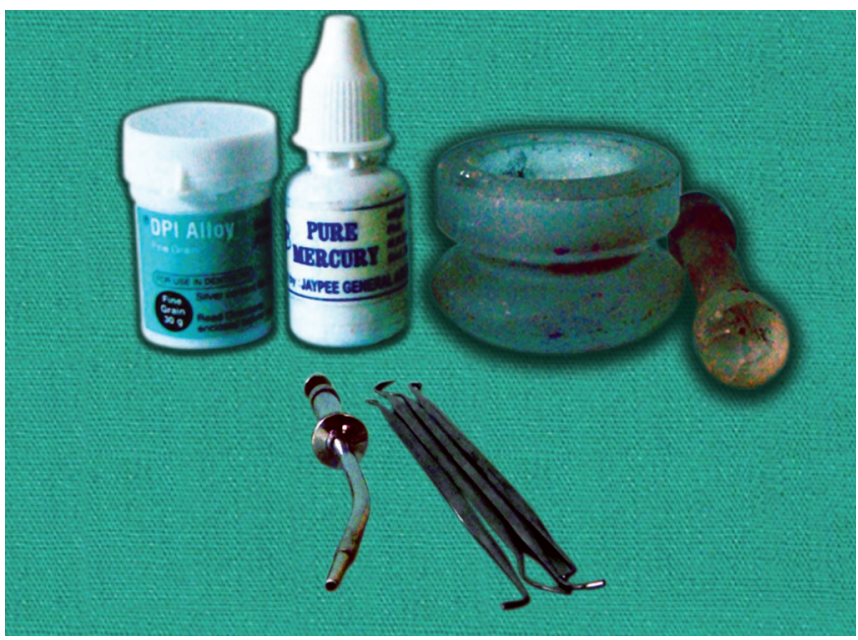

Fig. 3: Thermal insulator: Amalgam

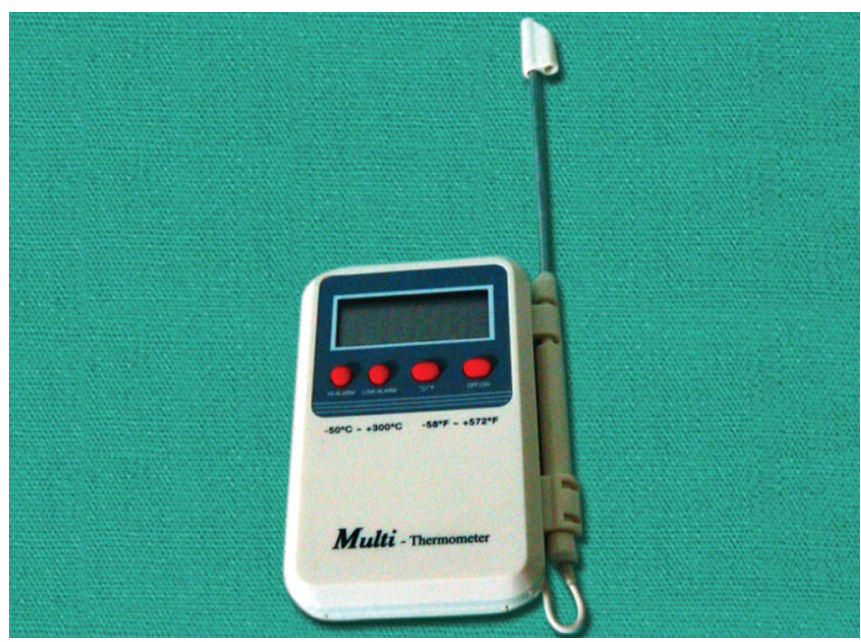

Fig. 2: Digital thermometer

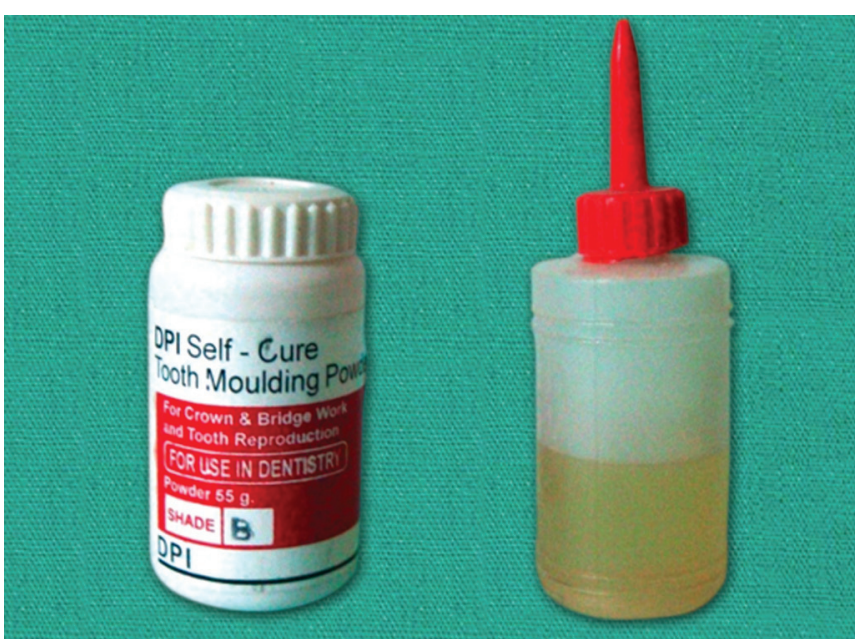

Fig. 4: Polymethyl methacrylate

Table 1: Mean temperature increase in pulp chamber during the polymerization of all the four tested provisional materials fabricated by direct technique

\begin{tabular}{|c|c|c|c|c|c|c|c|c|}
\hline \multirow[t]{3}{*}{ Materials } & \multicolumn{8}{|c|}{ Temperature increase } \\
\hline & \multirow[t]{2}{*}{$N$} & \multirow[t]{2}{*}{ Mean } & \multirow[t]{2}{*}{$\begin{array}{l}\text { Std. } \\
\text { deviation }\end{array}$} & \multirow[t]{2}{*}{ Std. error } & \multicolumn{2}{|c|}{$\begin{array}{l}\text { 95\% confidence interval for } \\
\text { mean }\end{array}$} & \multirow[t]{2}{*}{ Minimum } & \multirow[t]{2}{*}{ Maximum } \\
\hline & & & & & Lower bound & Upper bound & & \\
\hline PMMA & 20 & 38.785 & 0.9370 & 0.2095 & 38.346 & 39.224 & 37.1 & 39.8 \\
\hline PEMA & 20 & 37.794 & 0.5230 & 0.1170 & 37.549 & 38.039 & 36.9 & 38.5 \\
\hline PVEMA & 20 & 37.718 & 0.5105 & 0.1142 & 37.479 & 37.957 & 36.8 & 38.7 \\
\hline Bis-A composite & 20 & 35.882 & 0.5369 & 0.1201 & 35.630 & 36.133 & 34.6 & 36.6 \\
\hline Total & 80 & & & & & & & \\
\hline
\end{tabular}

Table 2: Comparison of mean temperature difference in pulp chamber during polymerization of four tested materials fabricated by direct technique

\begin{tabular}{llll}
\hline & \multicolumn{2}{c}{ Bonferroni multiple comparisons } & \\
\hline Group (I) & Group (J) & Mean difference (I-J) & -value \\
\hline PMMA & PEMA & $0.9910^{*}$ & $<0.0001$ \\
& PVEMA & $1.0670^{\star}$ & $<0.0001$ \\
& Bis-A composite & $2.9035^{*}$ & $<0.0001$ \\
PEMA & PVEMA & 0.0760 & 1.000 \\
& Bis-A composite & $1.9125^{*}$ & $<0.0001$ \\
PVEMA & Bis-A composite & $1.8365^{*}$ & $<0.0001$ \\
\hline
\end{tabular}

*Highly significant 


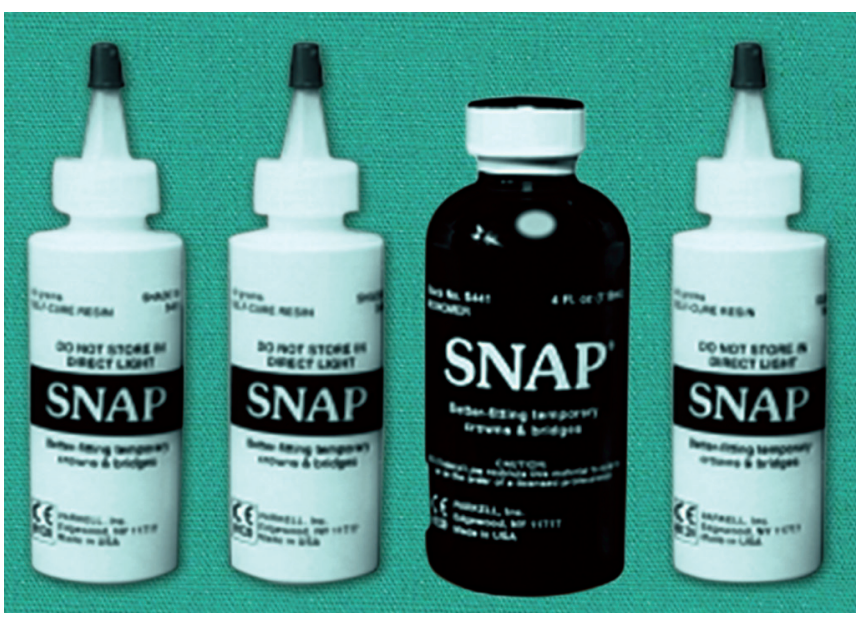

Fig. 5: Polyethyl methacrylate (SNAP)

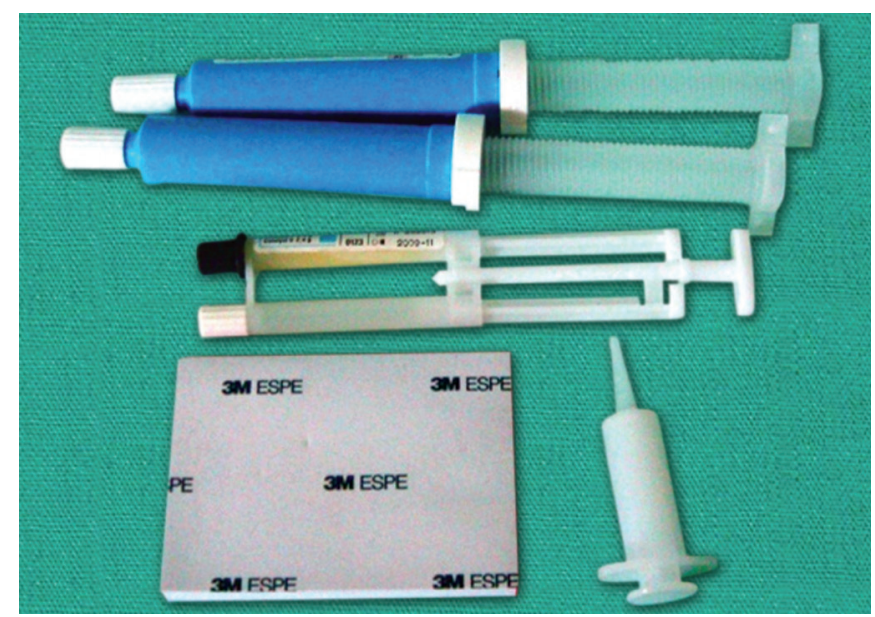

Fig. 7: Bis-acrylic composite (Protemp II)

When PEMA was compared with PVEMA, the statistical value was not significant and the mean difference was 0.760. On comparison of PEMA with bis-A composite, statistical analysis showed highly significant and there is statistical significance with a mean difference of 1.9125 . The statistical analysis showed highest significance, when PVEMA was compared with bis-A composite, and the mean difference was 1.8365 .

\section{DISCUSSION}

This in vitro study has compared the temperature increase in the pulp chamber of maxillary first premolar teeth placed in contact with four provisional restorative materials namely PMMA, PEMA, PVEMA and bis-A composite.

The results of the present study showed the mean value temperature obtained were $38.785^{\circ} \mathrm{C}, 37.794^{\circ} \mathrm{C}, 37.718^{\circ} \mathrm{C}$, and $35.882^{\circ} \mathrm{C}$ for PMMA, PEMA, PVEMA and bis-A composite respectively.

The statistical analysis showed highly significant when mean difference of PMMA was compared with mean difference of PEMA, PVEMA and bis-A composite and the mean differences were $0.9910,1.067$ and 2.903 respectively.

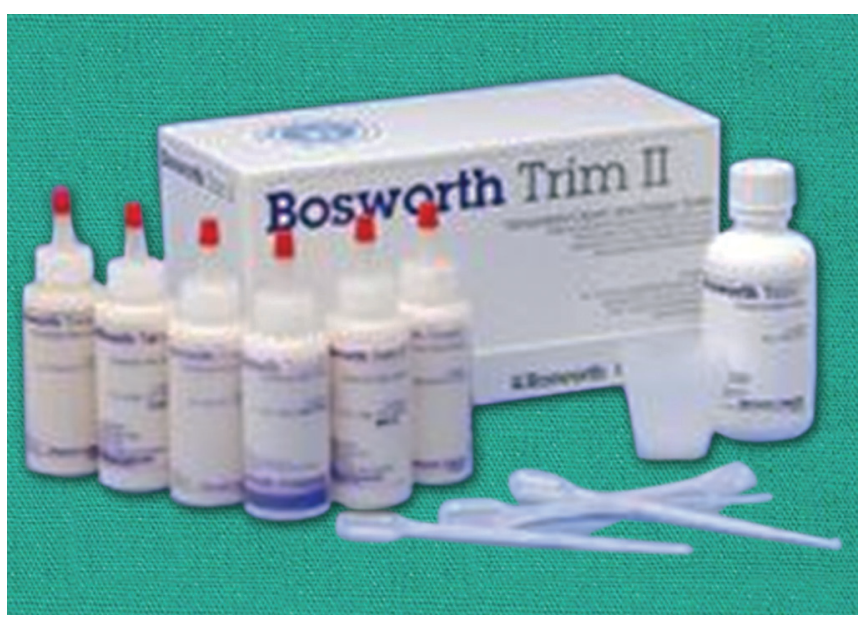

Fig. 6: Polyvinylethyl methacrylate

When PEMA was compared with PVEMA the statistical value was not significant and the mean difference was 0.760 . On comparison of PEMA with bis-A composite, statistical analysis showed highly significant and there is statistical significance with a mean difference of 1.9125 . The statistical analysis showed highest significance when PVEMA was compared with bis-A composite and the mean difference was 1.8365 .

A study conducted to assess the pulp response to external applied heat demonstrated that a temperature increase of $2.3^{\circ} \mathrm{C}$ produced minimum pulpal changes. These were confined to the odontoblasts that were next to the area of thermal injury. When the intrapulpal temperature was increased by $5.6^{\circ} \mathrm{C}$, a remarkable pulpal response was noted, including destruction of most of the odontoblasts, displacement of nuclei into the dentinal tubules, reduction of thickness of uncalcified predentin, denaturation of matrix, and destruction of Tomes' fibrils in the area of dentin immediately adjacent to the enamel that was touched by the instrument causing the thermal injury. However, after 56 days, $85 \%$ of the teeth subjected to this thermal trauma successfully overcome the inflammatory reaction. ${ }^{7}$

In the forgoing study, the intrapulpal changes resulting from the temperature increase during polymerization of the materials will range from 0 to $2.78^{\circ} \mathrm{C}$ for bis-A composite and PMMA respectively.

The rise in temperature of $2.78^{\circ} \mathrm{C}$ in case of PMMA though it is significantly high with that of bis-A composite, it is in the well tolerable limit. If the fabrication of provisional restorations by direct technique is preferred, precautionary measures must be used to minimize temperature increase of the tooth structure from the exothermic reactions of the resins. The temperature in the pulp could be reduced by using air and water coolant, by using controlled quantity of material, thermal insulator as petroleum jelly, use of room temperature water bath and removal of the temporary crown before the initial polymerization. ${ }^{4,8,9}$ Also, it could be done 
by use a putty matrix that this would act as a heat sink by dissipating exothermic heat. ${ }^{9}$

Temperature increase in the pulp for the forgoing study was ranging from 0 to $2.78^{\circ} \mathrm{C}$ for bis-A composite and PMMA respectively, whereas temperature rise in the pulp chamber was $1.7^{\circ} \mathrm{C}$ for both PEMA and PVEMA respectively.

In the referred study, the temperature rise in the pulp chamber of $2.3^{\circ} \mathrm{C}$ showed minimal intrapulpal changes. Only at $5.6^{\circ} \mathrm{C}$ increased temperature in the pulp showed remarkable pulpal changes, but these changes were recovered in 56 days. ${ }^{7}$ In the present study, PMMA showed temperature rise in pulp chamber was $2.78^{\circ} \mathrm{C}$, which was almost equal to the temperature $2.3^{\circ} \mathrm{C}$ and difference of $0.48^{\circ} \mathrm{C}$ is negligible.

All the materials used were in the safer limit. By comparing all the four material, bis-A composite showed least temperature rise in pulp chamber. The order of preference for the use of provisional restoration by direct technique would be bis-A composite, PVEMA, PEMA, PMMA with reference to rise in temperature in the pulp chamber when used in water bath with thermal insulator and controlled quantity of materials.

It should be noted that this is an in vitro study and therefore, has some limitations. Further in vivo studies are necessary to verify the results of this study.

\section{CONCLUSION}

The temperature increase in the pulp chamber during the fabrication of provisional resin crowns by direct method was recorded in this in vitro investigation.

All the materials used were in the safer limit. By comparing all the four materials, bis-A composite showed least temperature rise in pulp chamber. The order of preference for the use of provisional restoration by direct technique would be bis-A composite, PVEMA, PEMA and PMMA with reference to rise in temperature in the pulp chamber.

Therefore, clinical guidelines/implications are summarized below:

1. Removing the provisional restoration after the initial polymerization of resin. ${ }^{1}$

2. Using air/water spray: leaving the resin on the abutment teeth throughout the polymerization and spraying air/ water once the initial reaction has occurred. ${ }^{1}$

3. Room temperature water bath can be used to reduce the thermal insult to the pulp.
4. Thermal insulating medium as petroleum jelly or dental varnish can be used.

5. By using matrix material like addition silicone putty, this can act as a good heat sink.

6. Controlled quantity of materials also can reduce the insult to the pulp.

7. Use of an cement base or lining material can effectively reduce the intrapulpal temperature rise during light polymerization of composite resin..$^{10,11}$

\section{REFERENCES}

1. Manak E, Arora A. A comparative evaluation of temperature changes in the pulpal chamber during direct fabrication of provisional restorations: a in vitro study. J Indian Prosthodont Soc 2011 July-Sept;11(3):149-155.

2. Langeland K, Langeland LK. Pulp reaction to crown preparation, impression, temporary crown fixation, and permanent cementation. J Pros Dent 1965;15(1):129-143.

3. Jung-Yun HA, Sung-Hun KIM, Kyo-Han KIM, Tae-Yub KWON. Influence of the volumes of bis-acryl and poly (methyl methacrylate) resins on their exothermic behaviour during polymerization. Dent Mat J 2011;30(3):336-342.

4. Konstantinos M, Argiris P, Hiroshi H, Kiho K, Nikolaos K. Comparison of temperature increase in the pulp chamber during the polymerization of materials used for the direct fabrication of provisional restorations. J Prosthet Dent 2006;96:418-423.

5. Chadda AS, Verma PR, Nagpal A, Samra RK, Verma R, Katna $\mathrm{V}$. Evaluation of temperature changes in pulp chamber during direct temporization using different combinations of provisional crown and impression materials - an in vitro study. Indian J Dent Sci 2012 Dec;4(5):23-27.

6. Driscoll CF, Woolsey G, Ferguson WM. Comparison of exothermic release during polymerization of four materials used to fabricate interim restorations. J Prosthet Dent 1991;65:504-506.

7. Zach L, Cohen G. Pulp response to externally applied heat. Oral Surg Oral Med Oral Pathol 1965;19(4):515-530.

8. Tjan AHL, Dent DR, Grant BE, Godfrey MF. Temperature rise in pulp chamber during fabrication of provisional crowns. J Prosthet Dent 1989;62:622-626.

9. Kwon SJ, Park YJ, Jun SH, Ahn JS, Lee IB, Cho BH, Son HH, Seo DG. Thermal irritation of teeth during dental treatment procedures. Restor Dent Endod 2013;38(3):105-112.

10. Mousavinasab SM, Khoroushi M, Moharreri M. Temperature rise during primer, adhesive and composite resin photopolymerization of a low-shrinkage composite resin under caries like dentin lesions. Int Scholarly Resear Network Dent 2012;1-8.

11. Loureiro FH, et al. Comparison between two methods to evaluate temperature changes produced by composite light curing units and polymerization technique. Minerva Stomatol 2011 Oct; 60(10):501-508. 